\title{
OPTIMIZATION OF ROBUST HEADER COMPRESSION FOR AERONAUTICAL COMMUNICATION
}

\author{
R. Hermenier, C. Kissling, German Aerospace Center (DLR), Germany
}

\begin{abstract}
The ceaselessly growing requests for communication capacity in the aeronautical sector will soon result in an excess of the current Air Traffic Control (ATC) capacity limitations. To cope with this issue, current research activities, such as the EU FP7 SANDRA project, investigate new seamless IPv6based network solutions which integrate the different aeronautical services. These solutions will generate a significant protocol overhead due to all the required network functionalities. Within this paper, the focus is on the insertion of RObust Header Compression (ROHC) in the SANDRA architecture to reduce the protocol overheads. Performance evaluations for the usage of $\mathrm{ROHC}$ in the aeronautical context are provided and a strong emphasis of this work is put on a discussion on how ROHC parameters can be optimized for operational aeronautical communication.
\end{abstract}

\section{Introduction}

In the aeronautical sector, the demand for communication capacity is continuously growing. This increase is on one hand due to a significant growth in the number of passengers and thus flights but also due to the introduction of new aeronautical communication services with also increasing data volumes and a paradigm shift from analogue technologies towards digital ones. It is expected that the capacity limitations of current Air Traffic Control (ATC) systems are exceeded around 2020 [1] facing these challenges. Part of the currently ongoing research focuses on the development of new communication links, such as for instance satellite communication links in the ESA Iris program [2] or the terrestrial LDACS link [3]. Besides this, the integration of different link technologies into one large seamless aeronautical network will be a key component to serve the future communication demand.

The EU FP7 project SANDRA [4] focuses on the design of such a seamless network, integrating not only different communication links (by integration of radio technologies) and networks (such as ACARS, ATN/OSI, ATN/IPS), but also the different aeronautical service domains ATS, $\mathrm{AOC} / \mathrm{AAC}$ and $\mathrm{APC}$, and hybrid $\mathrm{Ku} / \mathrm{L}$ band antennas in a safe, high-performance and cost effective way. Figure 1 illustrates the different segments which SANDRA covers. Within the aircraft segment the key components are the Integrated Router (IR), the integrated modular radio (IMR), the integrated $\mathrm{Ku} / \mathrm{L}$ band satcom antennas and legacy equipment, consisting of legacy networks, antenna and modems. The transport segment consists of the different heterogeneous communication links, such as satellite or terrestrial links. On the ground side the integration of the different service provider networks with internet, public land mobile networks (PLMN) and the aeronautical telecommunication network (ATN) are covered.

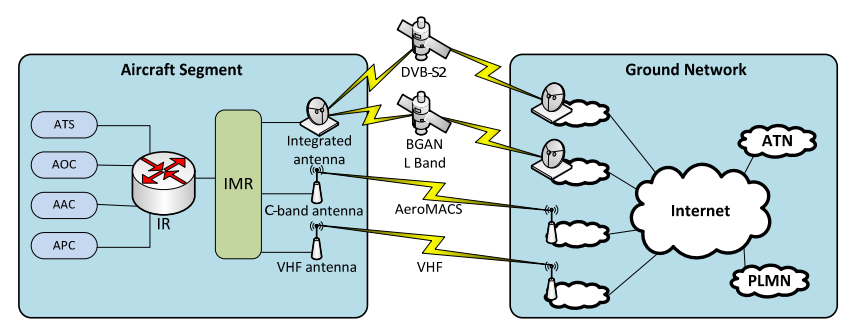

Figure 1: The SANDRA network architecture.

Within this paper we focus on operational aeronautical communication, i.e. Air Traffic Services (ATS) and Airline Operational Control (AOC). ATS comprises safety critical communication such as controller-pilot communication for directing airplanes through controlled airspace, separation of airplanes but includes also services such as position reporting. AOC services on the other hand have the purpose to exchange information between airplanes and their companies, AOC centers or operational staff at the airport. The different service applications which are foreseen to be deployed in the future aeronautical communication system are defined in the Communications Operation Concept and Requirements (COCR) document of EUROCONTROL and the FAA [5]. The COCR specifies (among other properties) for each service the message arrival rates and the sizes of the 
exchanged information. Here, we rely on other work which has dealt with deriving concrete traffic patterns out of the specifications given in the COCR, based on a detailed model of airplane movements and flight trajectories for the air traffic expected for the years 2013, 2020 and 2030 [6], [7], [8].

The high mobility of the considered aeronautical scenario, together with a very heterogeneous communication environment, consisting of different link technologies as well as access-, ground-, and onboard networks, creates the need for using sophisticated networking solutions. In addition, also security measures are required for protecting operational services. While AOC services may be encrypted and need to be authenticated, ATS services must not be encrypted since it is necessary that these services can always be monitored (but they anyway need to be authenticated). The different required mobility and security features introduce however also significant protocol overhead. For this reason the use of RObust Header Compression (ROHC) [9] techniques is of particular interest to reduce the network protocol overheads. ROHC is considered in the ICAO ATN IPS SARPS for IP implementation [10] and has, in other contexts, been shown to offer impressive performance also over links with large delays and prone to errors, such as is the case in satellite communication.

In the following, we provide an overview of the SANDRA networking concept, followed by an explanation how ROHC can be integrated into the SANDRA E2E protocol stack. The different options to integrate $\mathrm{ROHC}$ within the architecture will be investigated. In the second section, simulation results on the achievable compression gain when using aeronautical traffic will be provided. The core part of this work is presented in a third section, which focuses on the optimization of the ROHC usage. Based on a model that has been developed to investigate the behavior of ROHC for correlated wireless channels [11], [12], this paper shows how the ROHC key design parameters can be tuned to achieve a defined Quality of Service (QoS) depending on flight duration. The impact and consequences of modifying key design parameters of the ROHC protocol is discussed. Finally the future work and research directions conclude the paper.

\section{The SANDRA Networking Concept}

The SANDRA network concept relies on IPv6 and provides different networking functionalities in order to account for the particular demands of operational aeronautical communication.

First of all mobility needs to be provided to the airborne terminal by Mobile IPv6 due to the fast movement of airplanes. Additionally network mobility needs to be provided to the entire on-board subnetwork inside an aircraft, since several end systems are interconnected. Within SANDRA, the NEMO protocol [13] is used for this purpose with an additional SANDRA specific extension denoted SeNERO (Secure NEMO Routing Optimization) [14]. Since SANDRA aims at integration of legacy as well as future applications (based on the IPS) into one seamless network, a transition middleware or a (secure) dialogue service is foreseen in order to ensure a proper protocol translation towards the SANDRA IPv6 network.

Additionally, different transport layer protocols need to be available to support the heterogeneous requirements of the aeronautical services and the diverse link technologies. While TCP is a protocol mainly intended to provide back-compatibility and interoperability with existing networks, SANDRA also foresees the possibility to have tailored transport layer protocols, which provide reliable message delivery but without the overhead and complexity of TCP. For this purpose the Reliable Aeronautical Services Protocol (RASP) [15], which provides reliable end-to-end communication built on top of UDP was developed in SANDRA. Other services may not require a reliable transport layer connection at all, such as for instance position reports which are periodically sent and where directly a packet with updated information can be sent, instead of retransmitting a message with potentially already outdated information.

The ATS and AOC application services require furthermore the safe transmission of messages. In SANDRA, the provision of safety is based on the security mechanisms of the IPSec protocol, more specifically the use of IPSec Encapsulating Security Payloads (ESP) in tunnel mode. The message authentication and integrity check functionalities of IPSec are used for both ATS and AOC, while encryption is only applied to AOC for the aforementioned reasons. 


\section{Airborne Side}

\section{Ground Network}

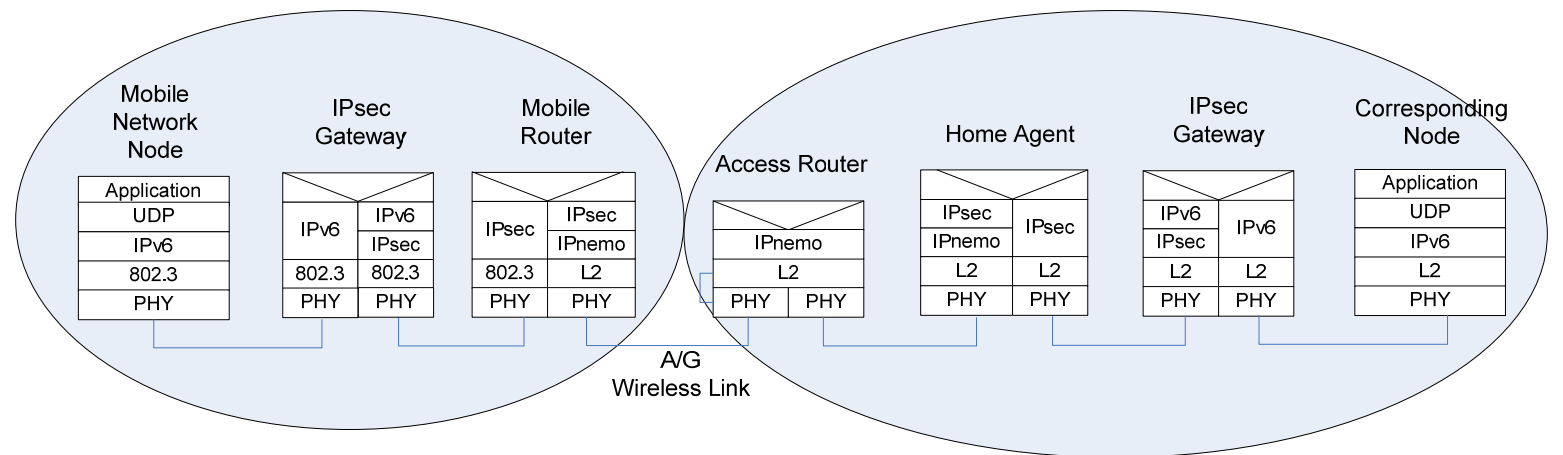

Figure 2: Functional architecture as used in SANDRA

\begin{tabular}{|c|c|c|c|c|}
\hline $\begin{array}{c}\text { IPv6 NEMO } \\
\text { Header }\end{array}$ & $\begin{array}{c}\text { IPv6 Sec } \\
\text { Header }\end{array}$ & IPv6 Header & UDP Header & Payload \\
\hline 40 B $\longrightarrow$ & 40 B \\
\hline
\end{tabular}

Figure 3: High level SANDRA IP Packet structure

Finally, a suitable QoS architecture needs to be put in place to ensure that the stringent latency and continuity requirements of the operational messages are met, that non-operational APC and AAC communication does not interfere with operational ATS and AOC and that the different physical entities and traversed networks can inter-operate smoothly and efficiently with each other.

Figure 2 shows the SANDRA network functionalities in a simplified protocol stack which illustrates how the airborne network entities connect to the ground network. The airborne applications connect via an on-board network with an airborne IPsecurity gateway which provides the IPSec security mechanisms for safety critical application services. The IPsec gateway is then connected to the airborne mobile router, which provides network mobility. The airborne network is connected to the ground network via an air-ground wireless link, where mobility is provided via a Home Agent (allowing route optimization as well). The correspondent node in the ground network is then reached after passing the IPsec gateway on ground.

In sum it can be concluded that the provision of all the aforementioned network functionalities generates a large amount of protocol overhead in addition to the message payload itself. Figure 3 illustrates the structure of a SANDRA IP packet, consisting of the IPv6 NEMO and IPv6 Sec, headers, the original IPv6 header itself, the transport protocol and the application payload.

Within the remainder of this work we investigate the possibility to reduce the protocol overhead, generated by all these functions by means of RObust Header Compression (ROHC) and analyze how ROHC can be used in the SANDRA network environment. Following this we provide performance evaluations for the usage of ROHC in the aeronautical context and a discussion on how ROHC parameters can be optimized for operational aeronautical communication.

\section{Introduction on ROHC}

Thanks to the innovative IP-based approach of the SANDRA concept, the significant amount of protocol overhead, generated by the architectural SANDRA features (such as security and mobility) can be reduced by using header compression techniques. In 2001, The IETF (Internet Engineering Task Force) has developed and standardized a header compression scheme entitled ROHC (RObust Header Compression [9]), which achieves very good performance over wireless links characterized by high loss ratio and long delay. Providing notably a high compression efficiency and high robustness, this technique has been designed to be used over scarce resources links, such as cellular or satellite links. $\mathrm{ROHC}$ is so effective that it has found its way in 
important wireless standards like HSPA and LTE [16], [17], [18] and is being currently proposed for the next generations of DVB RCS and DVB SH. On the other hand, only a limited amount of research has tried to investigate the performance and effectiveness of ROHC with aeronautical communication [19], although it is mentioned in the ICAO ATN IPS SARPS for IP implementation [10].

As many header compression schemes, ROHC works by removing the redundant information (static fields) contained in the protocol headers and transmits only the fields that may be subject to changes (dynamic fields). To further increase the compression efficiency and also the robustness, ROHC uses an encoding algorithm called W-LSB (Window-based Least Significant Bits) [9]. Based on a sliding window which contains information from previously compressed packets, this algorithm encodes only the difference between two consecutive headers and then transmits it. This mechanism ensures that the ROHC decompressor is still able to work properly even if up to SWW-1 packets are lost consecutively ([20], [21]), SWW being the sliding window width. [9], [11] and [12] show that this robustness can be increased up to $2 * S W W-2+p$ thanks to the use of the wraparound algorithm, where $p$ represents the offset with respect to the previously received field value. For the rest of this paper we will denote with $W=2 * S W W-2+p$ the maximal number of packets that can be deleted in a row while still obtaining a correct decompression of the next arriving packet.

Besides the W-LSB algorithm, the compression efficiency of ROHC is further expanded by the use of state machines at the compressor and decompressor sides. The former can operate in three states, namely the Initialization-Refresh state (IR state) in which static information are established, the First Order state (FO state) where the change pattern of dynamic fields are exchanged, and finally the Second Order state (SO state), in which only the encoding difference of dynamics fields (such as Sequence Number) for two consecutive headers is transferred. The ROHC compressor will then move upward from one state to the other to achieve high compression efficiency. If no feedback channel is available, the compressor will periodically transit downwards to lower state (FO or IR) based on two timeouts (FO Timeout (FOT) and IR Timeout (IRT) respectively) so as to ensure a regular refreshment of the information. Upon the correct reception of an IR packet, the compressor and decompressor initialize their context by storing information concerning the header. This context, used as basis for the compression and decompression, should always be synchronized between both compressor and decompressor. When no return channel is available for feedbacks, the synchronization is realized by the regular transmission of IR and FO packets to refresh the context.

All these mechanisms are implemented in the ROHC Unidirectional Mode (U-Mode), which will be our main focus for the rest of this paper. According to the standard, two other modes using feedback channels are also defined, Optimistic Mode (O-Mode) and Reliable Mode (R-Mode), but are considered out of scope for this work. More exhaustive insights on ROHC state machines and operational modes can be found in [9], [11], [12], [16], [20] and [21].

\section{ROHC within SANDRA}

As illustrated in Figure 3, there is a significant amount of overhead introduced by the SANDRA protocol stack. At first glance the ROHC mechanism could be inserted right after the network mobility encapsulation process when the IP packet is forwarded to the lower layers. However, this solution would require a $\mathrm{ROHC}$ decompressor in the Access Router so as to decode the network mobility IP header and route properly the packet (no routing possible with a compressed ROHC header). As the Access Router is handled by a terrestrial/satellite operator and is not a direct component of the SANDRA network, there is a limited possibility of deploying ROHC on it. Thus ROHC is only applied to compress the IPsec header as well as the original protocol headers (IPv6 and UDP in our example). It is therefore deployed on the Mobile Router (aircraft side) and Home Agent (ground side) before the network mobility mechanism as illustrated in Figure 4.

ROHC can compress a wide range of protocol headers such as, among others, IP, UDP or ESP. It is worth mentioning that when the IPsec profile (ESP) in ROHC is being used with an encryption algorithm other than NULL, the sub headers (IP, UDP, etc...) after the ESP header are encrypted and cannot 


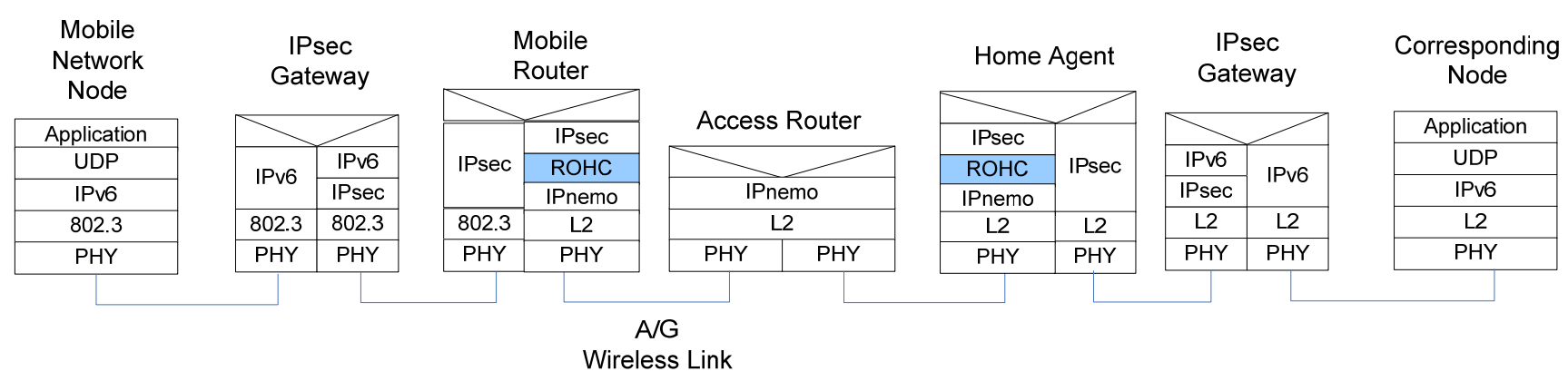

Figure 4: ROHC within an aeronautical IPS network architecture as defined in SANDRA

be compressed [9]. We will therefore assume the use of an encryption algorithm ESP NULL so that the content of the subsequent headers (IPv6 and UDP) is visible and thus compressible as is the case for ATS.

In the SANDRA network architecture ROHC can therefore be applied to the IPsec, IPv6 and UDP headers as illustrates in Figure 3.

\section{ROHC performance assessment for operational communication}

Using the available ROHC testbed at DLR premises based on an open-source implementation [22], this section provides compression gain results when ROHC is applied to operational aeronautical communication. [6], [7] and [8] provides different traffic patterns out of the COCR specifications. For this simulation, the ATS and AOC traffic pattern of a random 2 hours flight over Europe has been extracted. Table 1 illustrates the number of ATS and AOC messages exchanged during the considered flights, differentiating the forward (Ground to Aircraft) from the return link (Aircraft to Ground).

Table 1: ATS and AOC messages exchanged during the $2 \mathrm{H}$ flight considered

\begin{tabular}{|c|c|c|c|}
\hline & ATS & AOC & Total \\
\hline $\begin{array}{c}\text { Forward } \\
\text { Link }\end{array}$ & $\begin{array}{c}93 \\
\text { messages }\end{array}$ & $\begin{array}{c}86 \\
\text { messages }\end{array}$ & $\begin{array}{c}179 \\
\text { messages }\end{array}$ \\
\hline $\begin{array}{c}\text { Return } \\
\text { Link }\end{array}$ & $\begin{array}{c}92 \\
\text { messages }\end{array}$ & $\begin{array}{c}147 \\
\text { messages }\end{array}$ & $\begin{array}{c}239 \\
\text { messages }\end{array}$ \\
\hline Total & $\begin{array}{c}185 \\
\text { messages }\end{array}$ & $\begin{array}{c}233 \\
\text { messages }\end{array}$ & $\begin{array}{c}418 \\
\text { messages }\end{array}$ \\
\hline
\end{tabular}

For the purpose of this simulation, a specific case has been investigated where each of these 418 messages has been encapsulated in an IPv4 header (20 Bytes), compressed and then decoded at the decompressor side (this case is studied for transitions purposes). Figure 5 displays the testbed architecture. As the focus is only on the ROHC U-mode, no feedback channel was considered. It must also be noted that between the application and IP layer, a middleware may impact the size of the message coming from the application layer in the real SANDRA architecture. However this aspect is not considered here and the size of the messages at the application layer is considered to be the one at the IP layer.

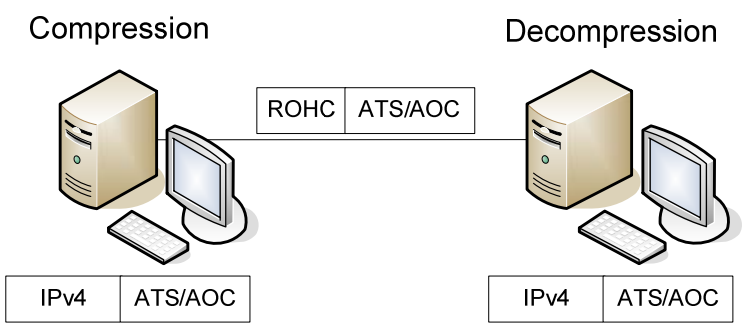

Figure 5: ROHC testbed

Furthermore it must be noticed that the size of some of the ATS/AOC messages are bigger than the MTU (1500 Bytes) of the testbed. Those messages have therefore been fragmented and encapsulated in different IPv4 packets so as to fit the MTU requirement. This applies to the following ATS services applications: COTRAC_Wilco (1613 Bytes, Forward Link), COTRAC_Interactive (1969 Bytes, Forward Link), FLIPINT (2763 Bytes, Return Link) as well as to the AOC service WXGRAPH (21077 Bytes, Forward Link). As a consequence, the overall number of IP packets exchanged after fragmentation increases from 179 original messages to 560 IP 
packets for the forward link and from 239 messages to 246 IP packets for the return link.

Simulation results are shown in Figure 6 and Figure 7, for the following ROHC configuration: IRT $=150, \mathrm{FOT}=80, \mathrm{~L}=3$ and $\mathrm{SWW}=14$ as suggested in [21], where $\mathrm{L}$ is the number of packets transmitted in IR and FO states.

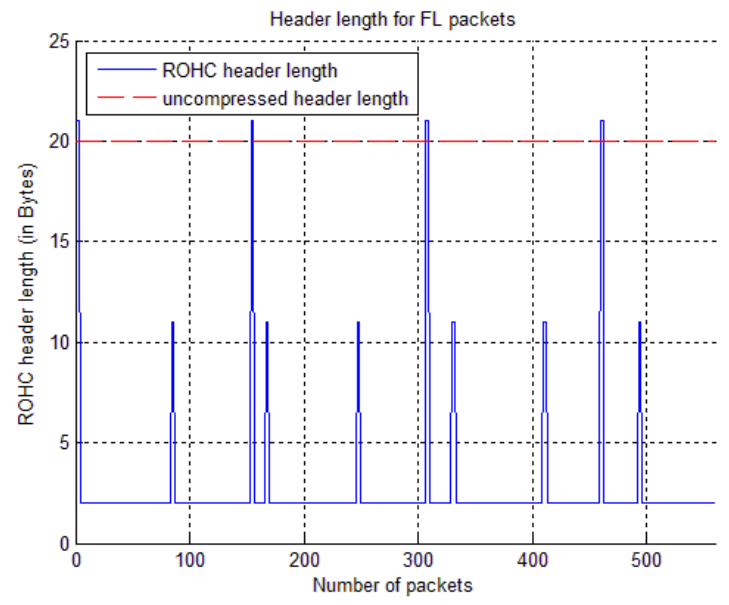

Figure 6: ROHC Header length (Forward Link)

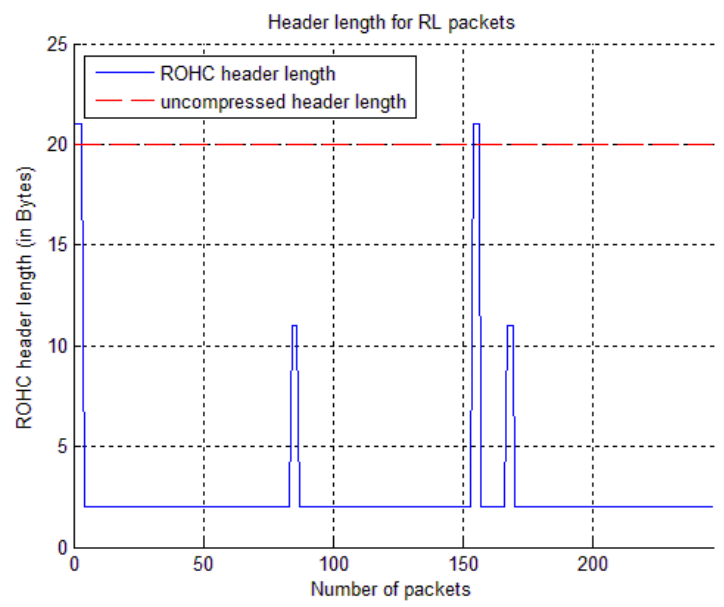

Figure 7: ROHC Header length (Return Link)

Table 2: Header and Overall Packet Size Gain

\begin{tabular}{|c|c|c|}
\hline & $\begin{array}{c}\text { Header Size } \\
\text { Gain }\end{array}$ & $\begin{array}{c}\text { Overall Packet } \\
\text { Size Gain }\end{array}$ \\
\hline Forward Link & $86.5 \%$ & $1.6 \%$ \\
\hline Return Link & $86.6 \%$ & $6.1 \%$ \\
\hline
\end{tabular}

Table 2 translates the results from the two previous figures into header and overall packet size gain. Those results illustrate that for the forward link, the high amount of fragmented packets (from 179 to 560) reduces the overall packet size gain significantly since many very large messages are transmitted, such as for WxGraph service, which provides weather forecast information to the aircraft. On the other hand this gain is higher for the return link due to the small payload size of the transmitted messages.

To better understand the impact of the different services (ATS and AOC), Table 3 and Table 4 show the header and overall size gains according to the ATS and AOC services, respectively.

Table 3: Header and Overall Packet Size Gain for ATS services

\begin{tabular}{|c|c|c|}
\hline ATS & $\begin{array}{c}\text { Header Size } \\
\text { Gain }\end{array}$ & $\begin{array}{c}\text { Overall Packet } \\
\text { Size Gain }\end{array}$ \\
\hline Forward Link & $86.4 \%$ & $3.5 \%$ \\
\hline Return Link & $85.5 \%$ & $3.1 \%$ \\
\hline
\end{tabular}

Table 4: Header and Overall Packet Size Gain for AOC services

\begin{tabular}{|c|c|c|}
\hline AOC & $\begin{array}{c}\text { Header Size } \\
\text { Gain }\end{array}$ & $\begin{array}{c}\text { Overall Packet } \\
\text { Size Gain }\end{array}$ \\
\hline Forward Link & $86.5 \%$ & $1.4 \%$ \\
\hline Return Link & $87.1 \%$ & $14.1 \%$ \\
\hline
\end{tabular}

Focusing on Table 4, the very high gain obtained for the return link (14.1\%) is due to the small payload size of the transmitted messages, whereas for the forward link, the WxGraph service considerably reduced the overall gain (1.4\%). For ATS services no major difference is noticeable between forward and return link as almost the same amount of ATS messages with no major variation of size is sent over both links.

Eventually it is worth recalling that the presented overall gains correspond solely to the achievable results with an IPv4 implementation for this specific selected flight. Further simulations with different traffic patterns would be required to draw exhaustive conclusions on the compression gain. The latter could be significantly improved when applying ROHC to the whole SANDRA protocol overhead (2 IPv6 and 1 UDP header) and not to a single IPv4 header. As presented in [19], ROHC allows a non- 
negligible bandwidth saving when implemented in the SANDRA architecture.

The previous simulations have been carried out without consideration of the arrival times but just on assuming consecutive transmission. The focus was therefore on the achievable compression rate. In reality ATS and AOC messages might be transmitted sporadically including long time period without data exchange. Using the same traffic pattern and ROHC configuration parameters as before, Figure 8 and Figure 9 illustrate the outcome of real-time simulations, showing the header length over time for both forward and return link, respectively.

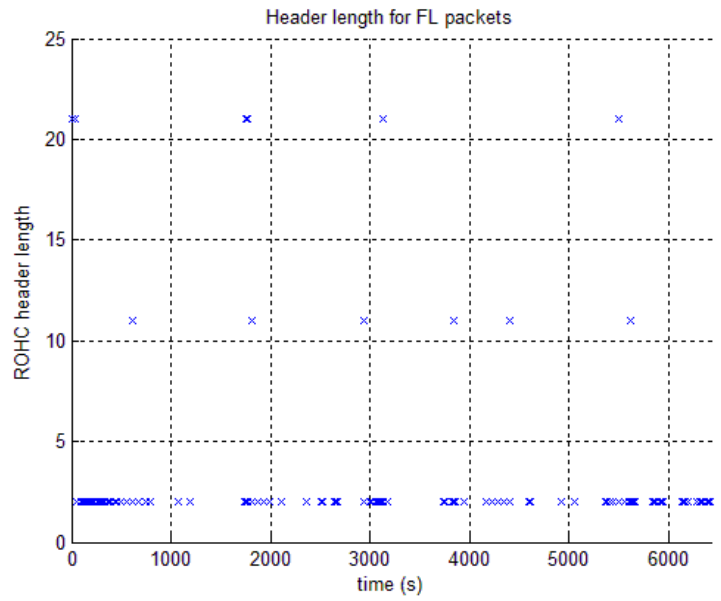

Figure 8: ROHC header length over time (Forward Link)

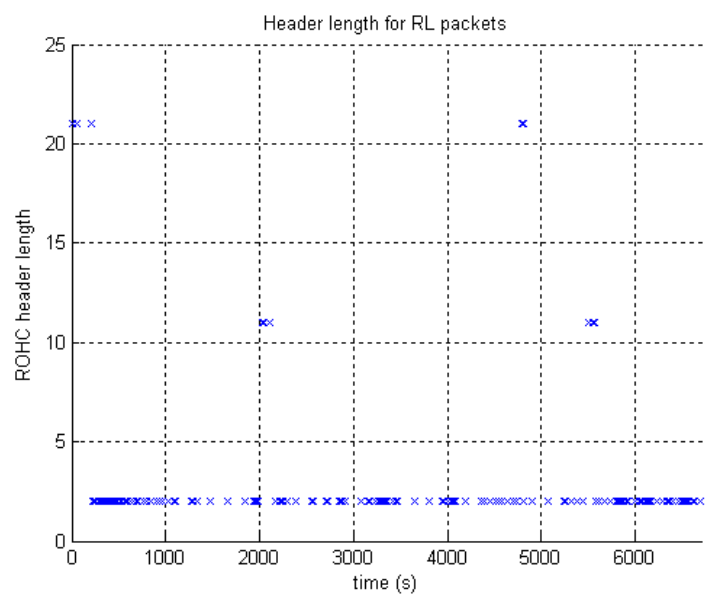

Figure 9: ROHC header length over time (Return Link)

As expected, the traffic pattern includes time intervals (up to 10 minutes) in which no transmission occurs. However this does not impact the behavior of
ROHC. Despite those transmission gaps, both contexts (compressor and decompressor) remain synchronized and the same compression performance as during the non-real-time simulations is achieved.

\section{Optimization of ROHC key parameters}

The previous simulations have been carried out with a specific configuration of the ROHC key parameters. This section discusses the possibility to optimize those parameters depending on the required needs.

\section{Scenario Definition}

The insertion of ROHC into a system may introduce additional losses called ROHC loss. The latter refers to the number of packets discarded due to ROHC. This situation may happen when the compressor and the decompressor have lost their context synchronization: packets are discarded until the correct reception of an IR uncompressed packet. Thus when integrating ROHC into a new architecture (such as SANDRA), it must be taken into account that additional loss might be generated by the use of this header compression technique. A natural question coming up is: what is the optimal configuration of ROHC key parameters so to minimize those losses and not worsen the overall QoS? [20] and [21] present research work on the optimization of those parameters for UMTS network. The next sections propose a theoretical approach to select the best configuration when ROHC is applied to operational aeronautical communication.

\section{Model explanation}

Based on deletion channels, [11] and [12] present an analytical model for ROHC U-mode that allows a proper tuning of ROHC key parameters depending on the required QoS. The erasure channel considered is a Gilbert-Elliott channel (two states Markov Chain) of average erasure probability $\varepsilon$ and average duration of a sequence of bad states $L_{B}$ (packet lost). In the aeronautical context, such a sequence of bad states may occur for long-haul flights reaching high latitudes and whose antenna (installed on the top of the aircraft) could not be in line-of-sight with the satellite as covered by the wing of the airplane (low elevation angle at high latitude). It could also happen during taxiing: the aircraft may be shadowed by buildings and not be in line-of-sight 
with the ATC control tower. Unless otherwise stated, the Gilbert-Elliott model parameters adopted are $\varepsilon=$ $2 \%$ and $L_{B}=5$ as suggested in [11]. Although these values are more suitable for terrestrial wireless link rather than aeronautical channel, their modification will not change the qualitative behavior of the system and therefore the conclusions drawn. Only numerical results such as the absolute header compression gain might change.

The proposed model links analytically the ROHC key parameters with the channel characteristics $\left(\mathrm{L}_{\mathrm{B}}\right.$ and $\varepsilon$ ) and shed the light into the achievable performance. Among the major ROHC parameters considered are the IR timeout (IRT) and $\mathrm{W}$ (as defined in the previous section). In this model, $\mathrm{L}$ has been set to 1 and the FO timeout is not considered. More exhaustive insights on this model can be found in [11] and [12].

\section{Theoretical Analysis}

The ROHC system must now be designed in such a way that the average erasure probability $\varepsilon$ is not significantly affected by the ROHC loss. Let us assume the following QoS:

$$
P_{\text {ROHC_Loss }} \leq 5 \% \cdot \varepsilon
$$

with $P_{\text {ROHC_Loss }}$ being the probability of having additional loss due to ROHC.

According to [11] and [12], the doublets (W, IRT) fulfilling our desired QoS can be derived, as illustrated in Figure 10. Table 5 presents the corresponding values for SWW.

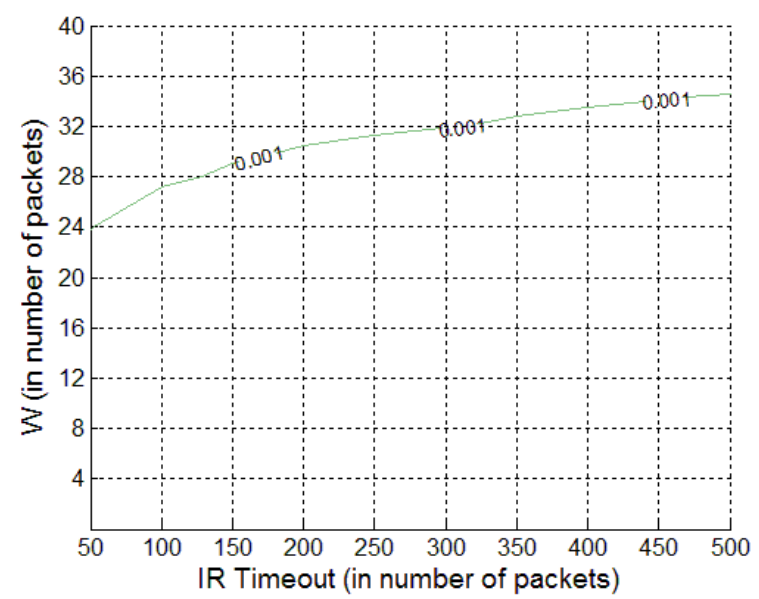

Figure 10: (W, IRT) fulfilling $P_{\text {ROHC_Loss }} \leq 5 \% \cdot \varepsilon$
Table 5: ROHC parameters fulfilling the QoS

\begin{tabular}{|c|c|c|c|c|c|c|}
\hline W & 24 & 25 & 27 & 29 & 31 & 33 \\
\hline SWW & 13 & 14 & 15 & 16 & 17 & 18 \\
\hline IRT & 50 & 70 & 90 & 150 & 250 & 350 \\
\hline
\end{tabular}

As specified in the previous section, [6], [7] and [8] provide traffic pattern statistics, such as an estimation of the average message arrival rate (ATS and AOC traffic) for different concentration of aircraft. Considering a high density of airplanes, Table 6 provides the average number of operational aeronautical messages received by an aircraft for different flight durations.

Table 6: Number of operational messages received

\begin{tabular}{|c|c|}
\hline Flight Duration (in hours) & $\begin{array}{c}\text { Number of ATS and AOC } \\
\text { messages received }\end{array}$ \\
\hline 1 & 346 \\
\hline 2 & 707 \\
\hline 3 & 1069 \\
\hline 4 & 1431 \\
\hline 5 & 1779 \\
\hline 6 & 2140 \\
\hline 7 & 2503 \\
\hline 8 & 2864 \\
\hline 9 & 3226 \\
\hline 10 & 3574 \\
\hline
\end{tabular}

Figure 11 illustrates the average header gain obtained considering the doublets (SWW, IRT) fulfilling the QoS from Table 5 and the different flight durations from Table 6. The ROHC packet sizes used to compute this gain are based on the values obtained from the simulation testbed using an IPv4 header only: 21 bytes for IR packets and 2 bytes for SO packets when SWW=14. However in the case where SWW > 14, a larger SO packet ( 3 bytes instead of 2) is used as more bits are necessary to encode the transmitted dynamic fields [21]. 


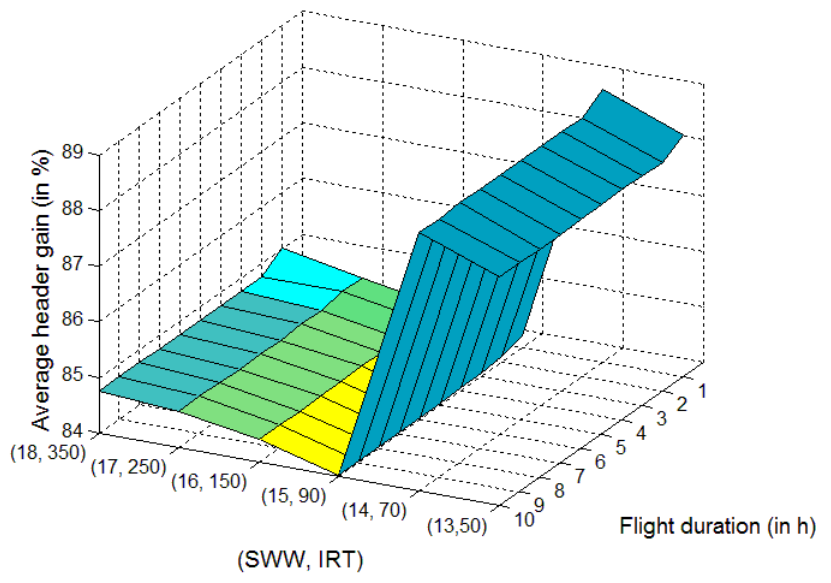

Figure 11: Average header gain

An immediate conclusion that can be drawn from Figure 11 is the uncorrelation of the compression efficiency with the flight duration, apart from very short flights where small changes can be observed. The average header gain is indeed not affected by the duration of a flight. Thus no ROHC parameter optimization is required for different flight durations. Furthermore it must be noted that the header gain is on average reduced when SWW $>14$ as larger SO packets are transmitted, as explained before. Moreover, for short flight duration (1h), the achieved gain is slightly higher than for longer flights (for a fixed (SWW, IRT)). This is mainly due to the fact that for such flights, few messages (and therefore few uncompressed IR packets) are sent, which leads to a higher compression gain. Finally Table 7 provides information on the Average Compressed header Length (ACL - as introduced by [21]) obtained for each doublet (SWW, IRT) fulfilling the desired QoS.

Table 7: ACL achieved for each doublet (SWW, IRT)

\begin{tabular}{|c|c|c|c|c|c|c|}
\hline SWW & 13 & 14 & 15 & 16 & 17 & 18 \\
\hline IRT & 50 & 70 & 90 & 150 & 250 & 350 \\
\hline $\begin{array}{c}\text { ACL } \\
\text { (bytes) }\end{array}$ & 2.38 & 2.27 & 3.2 & 3.12 & 3.07 & 3.05 \\
\hline
\end{tabular}

\section{Discussion on ROHC use for aeronautical communication}

The previous sub-section has shown that different configurations of the $\mathrm{ROHC}$ parameters could be adopted to fulfill the targeted QoS. The final decision for selecting the values to be used will depend on the needs for the final system. A trade-off between robustness and efficiency will have to be discussed to decide the most appropriate configuration of ROHC. One could favor for instance the efficiency by selecting a small SWW or on the other hand give advantage to the robustness and choose a low value of IRT (and the corresponding value for SWW based on the previous analysis).

Table 7 and Figure 11 show that changing from one configuration to another for an IPv4-only based system would not bring a significant improvement in terms of ACL (less than 1 byte gained) and average header gain (4\% in the best scenario), respectively. The same conclusion can be drawn when applying this analysis to the SANDRA protocol stack (compression of 2 IPv6 headers and 1 UDP header) as the achieved compression gains of two different configurations will only slightly differ. Furthermore, changing the ROHC configuration would require the transmission of additional uncompressed signaling packets to let the decompressor know about the new configuration, thus reducing the overall efficiency.

In an architecture as complex as the SANDRA one, a possible future evolution of ROHC would be to set a different configuration profile for each data link. As shown in Figure 1, the SANDRA system contains different data links with different characteristics. The AeroMACS and VDL2 links could be seen here as links requesting a higher robustness as they are transmitting safety critical information. Therefore a more robust scheme could be envisaged (lower IRT, larger SWW) as well as the $\mathrm{ROHC} \mathrm{O}-$ and R-modes as they provide feedback channels, ensuring a higher reliability of the system. Furthermore due to low RTT for those links, potential errors in the context could be corrected faster in case feedback is used (O- or R-mode), thus L could be selected as low as possible, as explained in [20]. Conversely satellite links are used here for passenger communication, which are non-safety related. A less robust but more efficient scheme could be foreseen (low SWW, large IRT), also considering that it would allow saving satellite capacity, and thus cost. In situations with a long RTT, a higher value of $\mathrm{L}$ would be beneficial as this would ensure communicating the updates with more confidence. 
Finally, a possible further extension could be to dynamically adjust SWW as link conditions are observed [16]. Including this feature in the ROHC configuration of each SANDRA data link would allow a more efficient use of ROHC, achieving higher compression rate during optimal link conditions.

\section{Conclusions}

Within this paper, we have shed the light on the achievable performance when ROHC is applied to operational aeronautical communication. Using real ATS/AOC traffic patterns, it has been shown that the flight duration and the sporadic transmission of information do no impact ROHC behavior and especially the compression efficiency.

As ROHC is a complex algorithm whose performance depends on the configuration of numerous key parameters, this work also presented a way to optimize those parameters based on a targeted QoS. Finally possible ROHC configurations based on link conditions have been discussed. Configuring ROHC often results in making a trade-off between robustness and efficiency.

At last, this work brings lots of opportunities for future work as on the one hand we foresee to run further simulations with different traffic patterns and on the other hand, deeper studies on the influence of other ROHC key parameters will be conducted to optimize the usage of $\mathrm{ROHC}$ for aeronautical communications.

\section{References}

[1] in Europe, A. C. F. A. R. European Aeronautics: A vision for 2020: Report of the Group of Personalities Office for Official Publications of the European Communities, 2001

[2] Morlet, C.; Ongaro, F.; Ricard, N. \& Santovincenzo, A. ESA Iris Programme: "Design options for the satellite communication sub-network of the European Air Traffic Management System", Proceedings of the 29th DASC, Salt Lake City (USA), Oct. 3-7, 2010.

[3] http://www.eurocontrol.int/communications/pub lic/standard page/LDACS.html

[4] Plass, S. "Seamless networking for aeronautical communications: One major aspect of the SANDRA concept", Aerospace and Electronic
Systems Magazine, IEEE, 27(9):21-27, September 2012

[5] Eurocontrol, F. Communications Operating Concept and Requirements for the Future Radio System (COCR) Eurocontrol/FAA, 2007.

[6] Rokitansky, C.-H.; Ehammer, M. \& Graupl, T. "Communication capacity assesment for the Iris satellite system", Proceedings of the 27th DASC, St. Paul, Minnesota (USA), Oct. 26-30, 2008.

[7] Morlet, C.; Ehammer, M.; Graupl, T. \& Rokitansky, C. "Characterisation of the data link communication air traffic for the European airspace" Proceedings of the 29th DASC, Salt Lake City (USA), Oct. 3-7, 2010.

[8] Gräupl, T. "Dimensioning requirements for the ANTARES ATM satellite data-link" Integrated Communications Navigation and Surveillance Conference (ICNS), May 11-13, 2010, 1 -22, Herndon (USA)

[9] Bormann, C.; et al., RFC 3095: "RObust Header Compression (ROHC): Framework and four profiles: RTP, UDP, ESP, and uncompressed", July 2001.

[10] ICAO, Aeronautical Telecommunication Network (ATN), "Manual for the ATN using IPS Standards and Protocols", Doc 9896, April 16, 2011, Draft Version 19

[11] Hermenier, R.; Rossetto, F. and Berioli, M.; "A Simple Analytical Model for RObust Header Compression in Correlated Wireless Links," in ISWCS, Aachen (Germany), 6-9 Nov 2011.

[12] Hermenier, R.; Rossetto, F. and Berioli, M.; "On the Behavior of RObust Header Compression Umode in Channels with Memory", submitted to IEEE Transactions on Wireless Communications, available at http://arxiv.org/abs/1303.4970

[13] Devarapalli, V.; Wakikawa, R.; Petrescu, A.; Thubert, P.; "Network Mobility (NEMO) Basic Support Protocol”, RFC 3963, January 2005.

[14] Bauer, C.; "NEMO Route Optimization with Strong Authentication for Aeronautical Communications" IEEE, 22nd IEEE Personal Indoor Mobile Radio Communications (PIMRC), 11-14 September 2011, Toronto.

[15] Muhammad, M.; Berioli, M.; (2011). Transport Protocol for Future Aeronautics, Future Aeronautical Communications, Dr. Simon Plass (Ed.), ISBN: 978-953-307-625-6, InTech, DOI: $10.5772 / 28455$ 
[16] Effnet, "The Concept of robust header compression, ROHC," White paper, February 2004.

[17] West, M.A.; Conroy, L.W.; Hancock, R.E.; Price, R. and Surtees, A.H.; "IP header and signalling compression for $3 \mathrm{G}$ systems," in $3 \mathrm{G}$ Mobile Communication Technologies, 2002, London (UK), May 2002.

[18] Rein, S.; Fitzek, F.H.P. and Reisslein, M. "Voice quality evaluation in wireless packet communication systems: a tutorial and performance results for ROHC," IEEE Wireless Commun. Mag., vol. 12, no. 1, pp. 60-67, Feb. 2005.

[19] Tordjman, T.; Luecke, O.; , "Evaluation of robust header compression for aeronautical operational data," Advanced Satellite Multimedia Systems Conference (ASMS) and 12th Signal Processing for Space Communications Workshop (SPSC), 2012 6th , vol., no., pp.308-315, 5-7 Sept. 2012, Baiona (Spain)

[20] Minaburo, A.; Nuaymi, L.; Kamal Deep Singh; Toutain, L., "Configuration and analysis of robust header compression in UMTS," Personal, Indoor and Mobile Radio Communications, 2003 (PIMRC 2003), 14th IEEE Proceedings on, pp. 2402- 2406 vol.3, 7-10 Sept. 2003, Beijing (China)

[21] Wang, B.; Schwefel, H.P.; Chua, K.C.; Kutka, R.; Schmidt, C.; , "On implementation and improvement of robust header compression in UMTS," Personal, Indoor and Mobile Radio Communications, 2002. The 13th IEEE International Symposium on , pp. 1151- 1155 vol.3, 15-18 Sept. 2002, Lisbon (Portugal)

[22] Barvaux, D.; "Robust Header Compression (ROHC) Library". Internet: https://launchpad.net/rohc

\section{Acknowledgments}

The research leading to these results has been partially funded by the European Community's Seventh Framework Program (FP7/2007-2013) under Grant Agreement $n^{\circ} 233679$. The SANDRA project is a Large Scale Integrating Project for the FP7 Topic AAT.2008.4.4.2 (Integrated approach to network centric aircraft communications for global aircraft operations). The project has 30 partners and started on 1st October 2009.

\section{Email Addresses}

Romain.hermenier@dlr.de

Christian.kissling@dlr.de

2013 Integrated Communications Navigation and Surveillance (ICNS) Conference

Apr 23-25, 2013 